\title{
Phataria unifascialis (Valvatida: Ophidiasteridae) from the Eastern Pacific: Redescription and skeletal morphology
}

\author{
Carolina Martín-Cao-Romero ${ }^{1 *}$, Francisco Alonso Solís-Marín², Alfredo Laguarda-Figueras ${ }^{2}$ \\ \& Blanca Estela Buitrón Sánchez ${ }^{3}$ \\ 1. Posgrado de Ciencias del Mar y Limnología, Instituto de Ciencias del Mar y Limnología (ICML), Universidad \\ Nacional Autónoma de México (UNAM), Apdo. Post. 70-305, México D.F. 04510, México; caromcr@gmail.com \\ 2. Laboratorio de Sistemática y Ecología de Equinodermos, Colección Nacional de Equinodermos "Dra. Ma. E. Caso M" \\ (CNE), Instituto de Ciencias del Mar y Limnología (ICML), Universidad Nacional Autónoma de México (UNAM), \\ Apdo. Post. 70-305, México D.F. 04510, México; fasolis@cmarl.unam.mx; laguarda@cmarl.unam.mx \\ 3. Departamento de Paleontología, Instituto de Geología, Universidad Nacional Autónoma de México, Circuito exterior \\ s/n, Col. Copilco, Del. Coyoacán, CP. 04500 México, D.F.; blancab@unam.mx \\ * Correspondence author
}

Received 13-I-2017. Corrected 19-VI-2017. Accepted 10-VII-2017.

\begin{abstract}
The starfish Phataria unifascialis is widely distributed in the eastern cost of the Pacific Ocean, found on rocky bottoms, at depths between 0 and $50 \mathrm{~m}$. The original description of $P$. unifascialis made by Gray in 1840 was brief and inaccurate in some important aspects, such as distribution area, plate arrangement and ambulacral spines. Here, we improve the description of $P$. unifascialis with SEM images and description of its mesodermal skeleton on the basis of a large sample. Rev. Biol. Trop. 65(Suppl. 1): S258-S271. Epub 2017 November 01 .
\end{abstract}

Key words: Asteroidea; Ophidiasteridae; Phataria unifascialis; redescription; SEM; Eastern Pacific.

Phataria unifascialis is a well-known starfish that inhabits the rocky bottoms of the Eastern Ocean Pacific shallow waters (Alvarado \& Solís-Marín, 2013). The starfish Phataria unifascialis belongs to the family Ophidiasteridae Verrill, 1870, which includes 28 valid genera, mostly shallow water, from tropical and subtropical regions. The species are a conspicuous feature of coral reef fauna. Asexual reproduction by fission occurs in a few species, but fertilization, planktotrophic or lecitothrophic larvae, and bipinnaria and/or brachiolaria stages are more common in the Ophidiasteridae family (Mortensen, 1921). The genus Phataria was proposed by Gray (1840) as a subgenus of the genus Linckia, and later was raised to genus by Sladen (1889). This genus differs from the others of the family Ophidiasteridae by having the papular areas in a longitudinal row or two in the radios, small granules in papular areas and big granules in the abactinal plates. Currently the genus Phataria has only two valid species: P. unifascialis (Gray, 1840) and Phataria mionactis Ziesenhenne, 1942.

Morgan and Cowles (1996) studied some aspects of the behaviour, physiology and demography of Phataria unifascialis, were examined to determine whether cool temperature was an important factor limiting this species' distribution in the northern regions of the Gulf of California. Individuals in the population at Bahia de Los Angeles, in the northern gulf, were of larger mean size but were significantly less abundant than at Loreto, a site in the southern gulf. Smaller size classes were also less abundant or missing in the Bahia de Los Angeles population, suggesting limited recruitment. Pyloric caecae and gonads were of similar size at both locations making it unlikely 
that food limitation or lack of reproductive potential was the limiting factor.

The population at Loreto contained a distinct reproductively-primed subgroup which was not observed at Bahia de Los Angeles. Activity of the species remained relatively constant with temperature down to $17^{\circ} \mathrm{C}$, the winter surface water temperature characteristic of Loreto, but dropped sharply at the $14^{\circ} \mathrm{C}$ water temperature characteristic of the Bahia de Los Angeles winter. Metabolic rates at these low temperatures were elevated over expected rates even though activity was reduced, suggesting metabolic stress. The species' moderate oxyregulating ability also disappeared at low temperature. Taken together, these data suggest that the low temperatures of the northern gulf may well be an important factor limiting this species' northern distribution in the Gulf (Morgan \& Cowles, 1996).
The purpose of the present study is to redescribe the species and its morphology based on several major ossicle types in the ophidiasterid.

\section{MATERIAL AND METHODS}

In this work 804 specimens were reviewed and they are deposited at the Colección Nacional de Equinodermos, "Dra. María Elena Caso Muñoz", (CNE, ICML, UNAM) and Los Angeles County Museum, Los Ángeles, California, USA (LACM) (Table 1). Organisms were observed using both an optic microscope (Olympus ${ }^{\circledR}$ SZX7) and scanning electron microscope (SEM) imaging (Laboratorio de Microscopía Electrónica de Barrido en el Instituto de Biología (IB), UNAM, Microscope JEOL JSM-6360LV). In order to observe the skeleton of $P$. unifascialis the skin

TABLE 1

Examined material of Phataria unifascialis and Phataria mionactis

CUADRO 1

Material examinado de Phataria unifascialis y Phataria mionactis

\begin{tabular}{|c|c|c|c|}
\hline & Catalogue number & $\begin{array}{l}\text { Number of } \\
\text { specimens }\end{array}$ & Locality \\
\hline Phataria unifascialis & ICML-UNAM 2.18.0 & 1 & $\begin{array}{l}\text { El Revolcadero, Acapulco, Guerrero, Mexico } \\
\left(16^{\circ} 47^{\prime} 45^{\prime \prime} \mathrm{N}, 99^{\circ} 51^{\prime} 17^{\prime \prime} \mathrm{W}\right)\end{array}$ \\
\hline Phataria unifascialis & ICML-UNAM 2.18.1 & 21 & $\begin{array}{l}\text { Isla Roca Partida, Baja California Sur, Mexico } \\
\left(24^{\circ} 10^{\prime} 00^{\prime} \mathrm{N}, 110^{\circ} 18^{\prime} 00^{\prime} \mathrm{W}\right)\end{array}$ \\
\hline Phataria unifascialis & ICML-UNAM 2.18.6 & 20 & $\begin{array}{l}\text { La Paz, Baja California Sur, Mexico } \\
\left(24^{\circ} 08^{\prime} 00^{\prime} \mathrm{N}, 110^{\circ} 23^{\prime} 00^{\prime \prime} \mathrm{W}\right)\end{array}$ \\
\hline Phataria unifascialis & ICML-UNAM 2.18 .8 & 6 & Isla Patos, Sonora, Mexico $\left(29^{\circ} 16^{\prime} 00^{\prime \prime} \mathrm{N}, 112^{\circ} 27^{\prime} 00^{\prime \prime} \mathrm{W}\right)$. \\
\hline Phataria unifascialis & ICML-UNAM 2.18 .12 & 2 & $\begin{array}{l}\text { Between Isla Ballena and Roca Partida, Baja California Sur, } \\
\text { Mexico }\left(24^{\circ} 20^{\prime} 00^{\prime} \mathrm{N}, 110^{\circ} 18^{\prime} 00^{\prime \prime} \mathrm{W}\right)\end{array}$ \\
\hline Phataria unifascialis & ICML-UNAM 2.18.14 & 1 & $\begin{array}{l}\text { Bahía Eclipse, Isla Roca Partida, Baja California Sur, Mexico } \\
\left(24^{\circ} 10^{\prime} 00^{\prime \prime} \mathrm{N}, 110^{\circ} 18^{\prime} 00^{\prime \prime} \mathrm{W}\right) .\end{array}$ \\
\hline Phataria unifascialis & ICML-UNAM 2.18.19 & 1 & $\begin{array}{l}\text { Bahía San Gabriel, Isla Espíritu Santo, Baja California Sur, } \\
\text { Mexico }\left(24^{\circ} 30^{\prime} 00^{\prime} \mathrm{N}, 110^{\circ} 21^{\prime} 00^{\prime} \mathrm{W}\right)\end{array}$ \\
\hline Phataria unifascialis & ICML-UNAM 2.18.23 & 32 & $\begin{array}{l}\text { La Paz, Baja California Sur, Mexico } \\
\left(24^{\circ} 08^{\prime} 00^{\prime} \mathrm{N}, 110^{\circ} 23^{\prime} 00^{\prime \prime} \mathrm{W}\right)\end{array}$ \\
\hline Phataria unifascialis & ICML-UNAM 2.18.24 & 28 & $\begin{array}{l}\text { La Paz, Baja California Sur, Mexico } \\
\left(24^{\circ} 08^{\prime} 00^{\prime \prime} \mathrm{N}, 110^{\circ} 23^{\prime} 00^{\prime} \mathrm{W}\right)\end{array}$ \\
\hline Phataria unifascialis & ICML-UNAM 2.18.26 & 3 & $\begin{array}{l}\text { Mazatlán, Sinaloa, Mexico } \\
\left(23^{\circ} 12^{\prime} 00^{\prime} \mathrm{N}, 106^{\circ} 25^{\prime} 00^{\prime \prime} \mathrm{W}\right)\end{array}$ \\
\hline Phataria unifascialis & ICML-UNAM 2.18.28 & 8 & $\begin{array}{l}\text { Cañón beach, Mazatlán, Sinaloa, Mexico } \\
\left(23^{\circ} 11^{\prime} 00^{\prime \prime} \mathrm{N}, 106^{\circ} 25^{\prime} 00^{\prime \prime} \mathrm{W}\right) \text {. }\end{array}$ \\
\hline
\end{tabular}


TABLE 1 (Continued)

\begin{tabular}{|c|c|c|c|}
\hline & Catalogue number & $\begin{array}{l}\text { Number of } \\
\text { specimens }\end{array}$ & Locality \\
\hline Phataria unifascialis & ICML-UNAM 2.18.29 & 11 & $\begin{array}{l}\text { Playa Norte, Mazatlán, Sinaloa, Mexico } \\
\left(23^{\circ} 14^{\prime} 13^{\prime \prime} \mathrm{N}, 106^{\circ} 26^{\prime} 45^{\prime \prime} \mathrm{W}\right) \text {. }\end{array}$ \\
\hline Phataria unifascialis & ICML-UNAM 2.18 .30 & 2 & $\begin{array}{l}\text { Playa Norte, Mazatlán, Sinaloa, Mexico } \\
\left(23^{\circ} 14^{\prime} 13^{\prime \prime} \mathrm{N}, 106^{\circ} 26^{\prime} 45^{\prime \prime} \mathrm{W}\right) \text {. }\end{array}$ \\
\hline Phataria unifascialis & ICML-UNAM 2.18 .31 & 2 & $\begin{array}{l}\text { Isla Chivos, Mazatlán, Sinaloa, Mexico } \\
\left(23^{\circ} 12^{\prime} 00^{\prime \prime} \mathrm{N}, 106^{\circ} 26^{\prime} 00^{\prime} \mathrm{W}\right)\end{array}$ \\
\hline Phataria unifascialis & ICML-UNAM 2.18 .32 & 1 & $\begin{array}{l}\text { The Crestón hill, off the lighthouse, Sinaloa, Mexico } \\
\left(23^{\circ} 10^{\prime} 47^{\prime \prime} \mathrm{N}, 106^{\circ} 26^{\prime} 00^{\prime \prime} \mathrm{W}\right) \text {. }\end{array}$ \\
\hline Phataria unifascialis & ICML-UNAM 2.18.33 & 9 & $\begin{array}{l}\text { The Crestón hill del lado Est. Mazatlán, Sinaloa, Mexico } \\
\left(23^{\circ} 10^{\prime} 57^{\prime \prime} \mathrm{N}, 106^{\circ} 25^{\prime} 51^{\prime \prime} \mathrm{W}\right) \text {. }\end{array}$ \\
\hline Phataria unifascialis & ICML-UNAM 2.18.34 & 23 & $\begin{array}{l}\text { Next to the Hermano del Sur, Sinaloa, Mexico } \\
\left(23^{\circ} 11^{\prime} 04^{\prime \prime} \mathrm{N}, 106^{\circ} 26^{\prime} 03^{\prime \prime} \mathrm{W}\right)\end{array}$ \\
\hline Phataria unifascialis & ICML-UNAM 2.18 .35 & 6 & $\begin{array}{l}\text { Next to Isla Venados, Sinaloa, Mexico } \\
\left(23^{\circ} 13^{\prime} 52^{\prime \prime} \mathrm{N}, 106^{\circ} 28^{\prime} 19^{\prime} \mathrm{W}\right)\end{array}$ \\
\hline Phataria unifascialis & ICML-UNAM 2.18 .36 & 13 & $\begin{array}{l}15 \text { m from Isla Venados, Sinaloa, Mexico } \\
\left(23^{\circ} 13^{\prime} 43^{\prime \prime} \mathrm{N}, 106^{\circ} 27^{\prime} 56^{\prime} \mathrm{W}\right)\end{array}$ \\
\hline Phataria unifascialis & ICML-UNAM 2.18 .37 & 15 & $\begin{array}{l}\text { Between Hermano del Norte and Hermano del Sur, Sinaloa, } \\
\text { Mexico }\left(23^{\circ} 11^{\prime} 10^{\prime} \text { N }, 106^{\circ} 26^{\prime} 30^{\prime} \text { W). }\right.\end{array}$ \\
\hline Phataria unifascialis & ICML-UNAM 2.18 .38 & 6 & $\begin{array}{l}\text { Proximities of Isla Cardones, Sinaloa, Mexico } \\
\left(23^{\circ} 10^{\prime} 52^{\prime \prime} \mathrm{N}, 106^{\circ} 24^{\prime} 10^{\prime} \mathrm{W}\right)\end{array}$ \\
\hline Phataria unifascialis & ICML-UNAM 2.18 .39 & 2 & $\begin{array}{l}\text { Between Isla Cardones and Isla de Chivos, Sinaloa, Mexico } \\
\left(23^{\circ} 10^{\prime} 41^{\prime \prime} \mathrm{N}, 106^{\circ} 24^{\prime} 25^{\prime} \mathrm{W}\right) \text {. }\end{array}$ \\
\hline Phataria unifascialis & ICML-UNAM 2.18 .40 & 7 & $\begin{array}{l}\text { Between Isla Cardones and Isla de Chivos, Sinaloa, Mexico } \\
\left(23^{\circ} 10^{\prime} 41^{\prime \prime} \mathrm{N}, 106^{\circ} 24^{\prime} 25^{\prime} \mathrm{W}\right) \text {. }\end{array}$ \\
\hline Phataria unifascialis & ICML-UNAM 2.18 .41 & 13 & $\begin{array}{l}\text { The Escollera, Sinaloa, Mexico } \\
\left(23^{\circ} 10^{\prime} 38^{\prime \prime} \mathrm{N}, 106^{\circ} 25^{\prime} 22^{\prime} \mathrm{W}\right)\end{array}$ \\
\hline Phataria unifascialis & ICML-UNAM 2.18 .42 & 7 & $\begin{array}{l}\text { Next to the Hermano del Sur, Sinaloa, Mexico } \\
\left(23^{\circ} 11^{\prime} 04^{\prime \prime} \mathrm{N}, 106^{\circ} 26^{\prime} 21^{\prime \prime} \mathrm{W}\right)\end{array}$ \\
\hline Phataria unifascialis & ICML-UNAM 2.18 .43 & 31 & $\begin{array}{l}\text { Cove beneath the tower at Paseo Centenario, Sinaloa, Mexico } \\
\left(23^{\circ} 11^{\prime} 07^{\prime \prime} \mathrm{N}, 106^{\circ} 25^{\prime} 41^{\prime \prime} \mathrm{W}\right) \text {. }\end{array}$ \\
\hline Phataria unifascialis & ICML-UNAM 2.18 .44 & 5 & $\begin{array}{l}\text { Next to the Hermano del Norte, Sinaloa, Mexico } \\
\left(23^{\circ} 11^{\prime} 22^{\prime \prime} \mathrm{N}, 106^{\circ} 26^{\prime} 15^{\prime \prime} \mathrm{W}\right)\end{array}$ \\
\hline Phataria unifascialis & ICML-UNAM 2.18.45 & 8 & $\begin{array}{l}\text { In the "entrance" of Hermano del Sur, Sinaloa, Mexico } \\
\left(23^{\circ} 10^{\prime} 56^{\prime} \text { N } 106^{\circ} 26^{\prime} 29^{\prime} \text { W). }\right.\end{array}$ \\
\hline Phataria unifascialis & ICML-UNAM 2.18 .46 & 4 & $\begin{array}{l}\text { Off Isla Venados, Sinaloa, Mexico } \\
\left(23^{\circ} 13^{\prime} 53^{\prime \prime} \mathrm{N}, 106^{\circ} 28^{\prime} 20^{\prime \prime} \mathrm{W}\right)\end{array}$ \\
\hline Phataria unifascialis & ICML-UNAM 2.18.47 & 11 & $\begin{array}{l}\text { Next to the south part of Isla de Lobos, Sinaloa, Mexico } \\
\left(23^{\circ} 13^{\prime} 42^{\prime \prime} \mathrm{N}, 106^{\circ} 27^{\prime} 54^{\prime \prime} \mathrm{W}\right) \text {. }\end{array}$ \\
\hline Phataria unifascialis & ICML-UNAM 2.18.48 & 1 & $\begin{array}{l}\text { In the "entrance" of Isla de Chivos, Sinaloa, Mexico } \\
\left(23^{\circ} 10^{\prime} 34^{\prime \prime} \mathrm{N}, 106^{\circ} 25^{\prime} 06^{\prime \prime} \mathrm{W}\right) \text {. }\end{array}$ \\
\hline Phataria unifascialis & ICML-UNAM 2.18 .49 & 3 & $\begin{array}{l}\text { Off the radio tower, Mazatlán, Sinaloa, Mexico } \\
\left(23^{\circ} 11^{\prime} 08^{\prime \prime} \mathrm{N}, 106^{\circ} 25^{\prime} 43^{\prime \prime} \mathrm{W}\right)\end{array}$ \\
\hline Phataria unifascialis & ICML-UNAM 2.18 .50 & 3 & $\begin{array}{l}\text { Two meters away of Hermano del Sur, Sinaloa, Mexico } \\
\left(23^{\circ} 10^{\prime} 51^{\prime \prime} \mathrm{N}, 106^{\circ} 26^{\prime} 39^{\prime \prime} \mathrm{W}\right) \text {. }\end{array}$ \\
\hline Phataria unifascialis & ICML-UNAM 2.18 .51 & 9 & $\begin{array}{l}\text { Off Isla Venados, Sinaloa, Mexico } \\
\left(23^{\circ} 13^{\prime} 44^{\prime \prime} \mathrm{N}, 106^{\circ} 28^{\prime} 17^{\prime} \mathrm{W}\right)\end{array}$ \\
\hline Phataria unifascialis & ICML-UNAM 2.18 .52 & 9 & $\begin{array}{l}\text { Off Isla Venados, Sinaloa, Mexico } \\
\left(23^{\circ} 13^{\prime} 53^{\prime \prime} \mathrm{N}, 106^{\circ} 28^{\prime} 20^{\prime} \mathrm{W}\right)\end{array}$ \\
\hline Phataria unifascialis & ICML-UNAM 2.18 .53 & 7 & $\begin{array}{l}\text { North cove of Isla de los Pájaros, Sinaloa, Mexico } \\
\left(23^{\circ} 15^{\prime} 41^{\prime \prime} \mathrm{N}, 106^{\circ} 28^{\prime} 46^{\prime} \mathrm{W}\right) \text {. }\end{array}$ \\
\hline
\end{tabular}


TABLE 1 (Continued)

\begin{tabular}{|c|c|c|c|}
\hline & Catalogue number & $\begin{array}{l}\text { Number of } \\
\text { specimens }\end{array}$ & Locality \\
\hline Phataria unifascialis & ICML-UNAM 2.18 .54 & 30 & $\begin{array}{l}\text { Cove between the Isla de Chivos and Isla Venados, Sinaloa, } \\
\text { Mexico }\left(23^{\circ} 13^{\prime} 44^{\prime \prime} \mathrm{N}, 106^{\circ} 27^{\prime} 56^{\prime \prime} \mathrm{W}\right) \text {. }\end{array}$ \\
\hline Phataria unifascialis & ICML-UNAM 2.18.55 & 25 & $\begin{array}{l}\text { Isla Venados, one side towards Isla Pájaros, Sinaloa, Mexico } \\
\left(23^{\circ} 14^{\prime} 34^{\prime \prime} \mathrm{N}, 106^{\circ} 28^{\prime} 20^{\prime \prime} \mathrm{W}\right) \text {. }\end{array}$ \\
\hline Phataria unifascialis & ICML-UNAM 2.18 .56 & 10 & $\begin{array}{l}60 \mathrm{~m} \text { to the east of Isla Cardones, Sinaloa, Mexico, Mexico } \\
\left(23^{\circ} 10^{\prime} 42^{\prime \prime} \mathrm{N}, 106^{\circ} 24^{\prime} 12^{\prime \prime} \mathrm{W}\right)\end{array}$ \\
\hline Phataria unifascialis & ICML-UNAM 2.18.57 & 37 & $\begin{array}{l}70 \mathrm{~m} \text { to the southwest of Isla de Venados, Sinaloa, Mexico } \\
\left(23^{\circ} 14^{\prime} 01^{\prime \prime} \mathrm{N}, 106^{\circ} 27^{\prime} 57^{\prime \prime} \mathrm{W}\right)\end{array}$ \\
\hline Phataria unifascialis & ICML-UNAM 2.18.58 & 20 & $\begin{array}{l}\text { Noth part of Isla de los Pájaros, Sinaloa, Mexico } \\
\left(23^{\circ} 15^{\prime} 40^{\prime \prime} \mathrm{N}, 106^{\circ} 39^{\prime} 12^{\prime \prime} \mathrm{W}\right)\end{array}$ \\
\hline Phataria unifascialis & ICML-UNAM 2.18 .59 & 38 & $\begin{array}{l}100 \text { m west of Isla Cardones, Sinaloa, Mexico } \\
\left(23^{\circ} 10^{\prime} 43^{\prime \prime} \mathrm{N}, 106^{\circ} 24^{\prime} 25^{\prime} \mathrm{W}\right)\end{array}$ \\
\hline Phataria unifascialis & ICML-UNAM 2.18.60 & 1 & $\begin{array}{l}80 \text { m southeast of Isla de Chivos, Sinaloa, Mexico } \\
\left(23^{\circ} 10^{\prime} 42^{\prime \prime} \mathrm{N}, 106^{\circ} 24^{\prime} 48^{\prime} \mathrm{W}\right) \text {. }\end{array}$ \\
\hline Phataria unifascialis & ICML-UNAM 2.18.61 & 9 & $\begin{array}{l}\text { East of Isla Pájaros, Sinaloa, Mexico } \\
\left(23^{\circ} 15^{\prime} 13^{\prime \prime} \mathrm{N}, 106^{\circ} 28^{\prime} 32^{\prime \prime} \mathrm{W}\right)\end{array}$ \\
\hline Phataria unifascialis & ICML-UNAM 2.18 .62 & 9 & $\begin{array}{l}\text { Between Isla Venados and Isla de Lobos, Sinaloa, Mexico } \\
\left(23^{\circ} 15^{\prime} 13^{\prime \prime} \mathrm{N}, 106^{\circ} 28^{\prime} 32^{\prime} \mathrm{W}\right) \text {. }\end{array}$ \\
\hline Phataria unifascialis & ICML-UNAM 2.18.63 & 1 & $\begin{array}{l}\text { Next to Hermano del Sur, Sinaloa, Mexico } \\
\left(23^{\circ} 11^{\prime} 06^{\prime \prime} \mathrm{N}, 106^{\circ} 26^{\prime} 19^{\prime \prime} \mathrm{W}\right)\end{array}$ \\
\hline Phataria unifascialis & ICML-UNAM 2.18 .64 & 10 & $\begin{array}{l}\text { Next to Isla de Lobos, Sinaloa, Mexico } \\
\left(23^{\circ} 13^{\prime} 47^{\prime \prime} \mathrm{N}, 106^{\circ} 28^{\prime} 19^{\prime \prime} \mathrm{W}\right)\end{array}$ \\
\hline Phataria unifascialis & ICML-UNAM 2.18.65 & 2 & $\begin{array}{l}\text { Next to Isla de Chivos, Sinaloa, Mexico } \\
\left(23^{\circ} 10^{\prime} 54^{\prime \prime} \mathrm{N}, 106^{\circ} 24^{\prime} 48^{\prime \prime} \mathrm{W}\right)\end{array}$ \\
\hline Phataria unifascialis & ICML-UNAM 2.18.66 & 4 & $\begin{array}{l}\text { Between Isla Venados and Isla de Lobos, Sinaloa, Mexico } \\
\left(23^{\circ} 13^{\prime} 43^{\prime \prime} \mathrm{N}, 106^{\circ} 27^{\prime} 58^{\prime \prime} \mathrm{W}\right) \text {. }\end{array}$ \\
\hline Phataria unifascialis & ICML-UNAM 2.18.67 & 5 & $\begin{array}{l}\text { Next to Isla Venados, Sinaloa, Mexico } \\
\left(23^{\circ} 14^{\prime} 06^{\prime \prime} \mathrm{N}, 106^{\circ} 27^{\prime} 57^{\prime \prime} \mathrm{W}\right)\end{array}$ \\
\hline Phataria unifascialis & ICML-UNAM 2.18 .68 & 4 & $\begin{array}{l}\text { Southwest to Isla de Pájaros, Sinaloa, Mexico } \\
\left(23^{\circ} 15^{\prime} 12^{\prime \prime} \mathrm{N}, 106^{\circ} 29^{\prime} 03^{\prime} \mathrm{W}\right) \text {. }\end{array}$ \\
\hline Phataria unifascialis & ICML-UNAM 2.18.69 & 8 & $\begin{array}{l}\text { Southwest of Isla Venados, Sinaloa, Mexico } \\
\left(23^{\circ} 13^{\prime} 53^{\prime \prime} \mathrm{N}, 106^{\circ} 27^{\prime} 50^{\prime \prime} \mathrm{W}\right) \text {. }\end{array}$ \\
\hline Phataria unifascialis & ICML-UNAM 2.18 .70 & 2 & $\begin{array}{l}\text { Southeast of Isla Cardones, Sinaloa, Mexico } \\
\left(23^{\circ} 10^{\prime} 43^{\prime \prime} \mathrm{N}, 106^{\circ} 24^{\prime} 12^{\prime \prime} \mathrm{W}\right) \text {. }\end{array}$ \\
\hline Phataria unifascialis & ICML-UNAM 2.18 .71 & 12 & $\begin{array}{l}\text { Northeast of Isla Pájaros, Sinaloa, Mexico } \\
\left(23^{\circ} 15^{\prime} 39^{\prime \prime} \mathrm{N}, 106^{\circ} 28^{\prime} 37^{\prime \prime} \mathrm{W}\right) \text {. }\end{array}$ \\
\hline Phataria unifascialis & ICML-UNAM 2.18 .72 & 10 & $\begin{array}{l}\text { West of Isla Cardones, Sinaloa, Mexico } \\
\left(23^{\circ} 10^{\prime} 40^{\prime \prime} \mathrm{N}, 106^{\circ} 24^{\prime} 28^{\prime \prime} \mathrm{W}\right)\end{array}$ \\
\hline Phataria unifascialis & ICML-UNAM 2.18 .73 & 1 & $\begin{array}{l}\text { Next to Isla Cardones, Sinaloa, Mexico } \\
\left(23^{\circ} 10^{\prime} 43^{\prime \prime} \mathrm{N}, 106^{\circ} 24^{\prime} 23^{\prime \prime} \mathrm{W}\right) \text {. }\end{array}$ \\
\hline Phataria unifascialis & ICML-UNAM 2.18 .74 & 1 & $\begin{array}{l}\text { East of Isla de Lobos, Sinaloa, Mexico } \\
\left(23^{\circ} 13^{\prime} 38^{\prime \prime} \mathrm{N}, 106^{\circ} 27^{\prime} 57^{\prime \prime} \mathrm{W}\right)\end{array}$ \\
\hline Phataria unifascialis & ICML-UNAM 2.18.75 & 1 & $\begin{array}{l}\text { In the "entrance" of Isla de Lobos, Sinaloa, Mexico } \\
\left(23^{\circ} 11^{\prime} 21^{\prime} \text { N }, 106^{\circ} 28^{\prime} 13^{\prime \prime} \mathrm{W}\right) \text {. }\end{array}$ \\
\hline Phataria unifascialis & ICML-UNAM 2.18 .76 & 4 & $\begin{array}{l}\text { Playa Norte, Mazatlán, Sinaloa, Mexico } \\
\left(23^{\circ} 14^{\prime} 13^{\prime \prime} \mathrm{N}, 106^{\circ} 26^{\prime} 45^{\prime \prime} \mathrm{W}\right)\end{array}$ \\
\hline Phataria unifascialis & ICML-UNAM 2.18 .77 & 15 & $\begin{array}{l}\text { Next to Isla de la Piedra, Sinaloa, Mexico } \\
\left(23^{\circ} 11^{\prime} 10^{\prime \prime} \mathrm{N}, 106^{\circ} 24^{\prime} 18^{\prime \prime} \mathrm{W}\right)\end{array}$ \\
\hline Phataria unifascialis & ICML-UNAM 2.18.78 & 11 & $\begin{array}{l}\text { La Escollera, Sinaloa, Mexico } \\
\left(23^{\circ} 10^{\prime} 50^{\prime \prime} \mathrm{N}, 106^{\circ} 25^{\prime} 30^{\prime \prime} \mathrm{W}\right)\end{array}$ \\
\hline
\end{tabular}


TABLE 1 (Continued)

\begin{tabular}{|c|c|c|c|}
\hline & Catalogue number & $\begin{array}{l}\text { Number of } \\
\text { specimens }\end{array}$ & Locality \\
\hline Phataria unifascialis & ICML-UNAM 2.18 .79 & 2 & $\begin{array}{l}\text { Between Isla de la Piedra and Isla de Chivos, Sinaloa, Mexico } \\
\left(23^{\circ} 10^{\prime} 56^{\prime \prime} \mathrm{N}, 106^{\circ} 24^{\prime} 39^{\prime \prime} \mathrm{W}\right) \text {. }\end{array}$ \\
\hline Phataria unifascialis & ICML-UNAM 2.18 .80 & 9 & $\begin{array}{l}\text { Next to Isla Cardones, Sinaloa, Mexico } \\
\left(23^{\circ} 10^{\prime} 42^{\prime \prime} \mathrm{N}, 106^{\circ} 24^{\prime} 13^{\prime \prime} \mathrm{W}\right)\end{array}$ \\
\hline Phataria unifascialis & ICML-UNAM 2.18 .81 & 7 & $\begin{array}{l}\text { Next to Isla Cardones, Sinaloa, Mexico } \\
\left(23^{\circ} 10^{\prime} 45^{\prime \prime} \mathrm{N}, 106^{\circ} 24^{\prime} 13^{\prime \prime} \mathrm{W}\right)\end{array}$ \\
\hline Phataria unifascialis & ICML-UNAM 2.18 .87 & 1 & $\begin{array}{l}\text { Next to Isla Venados, Sinaloa, Mexico } \\
\left(23^{\circ} 14^{\prime} 21^{\prime \prime} \mathrm{N}, 106^{\circ} 28^{\prime} 01^{\prime} \mathrm{W}\right)\end{array}$ \\
\hline Phataria unifascialis & ICML-UNAM 2.18.95 & 2 & $\begin{array}{l}\text { Isla la Ballena, Baja California Sur, Mexico } \\
\left(24^{\circ} 25^{\prime} 00^{\prime \prime} \mathrm{N}, 110^{\circ} 20^{\prime} 00^{\prime \prime} \mathrm{W}\right) \text {. }\end{array}$ \\
\hline Phataria unifascialis & ICML-UNAM 2.18.96 & 1 & $\begin{array}{l}\text { Los Islotes, Baja California Sur, Mexico } \\
\left(24^{\circ} 36^{\prime} 00^{\prime \prime} \mathrm{N}, 110^{\circ} 23^{\prime} 00^{\prime \prime} \mathrm{W}\right)\end{array}$ \\
\hline Phataria unifascialis & ICML-UNAM 2.18.97 & 1 & $\begin{array}{l}\text { La Gaviota, La Paz, Baja California Sur, Mexico } \\
\left(24^{\circ} 17^{\prime} 13^{\prime \prime} \mathrm{N}, 110^{\circ} 20^{\prime} 34^{\prime \prime} \mathrm{W}\right)\end{array}$ \\
\hline Phataria unifascialis & ICML-UNAM 2.18.101 & 6 & $\begin{array}{l}\text { Bahía San Gabriel, Isla Espíritu Santo, Baja California Sur, } \\
\text { Mexico }\left(24^{\circ} 30^{\prime} 00^{\prime \prime} \mathrm{N}, 110^{\circ} 21^{\prime} 00^{\prime} \mathrm{W}\right) \text {. }\end{array}$ \\
\hline Phataria unifascialis & ICML-UNAM 2.18.102 & 1 & $\begin{array}{l}\text { Puerto Escondido, Baja California Sur, Mexico } \\
\left(25^{\circ} 50^{\prime} 00^{\prime \prime} \mathrm{N}, 111^{\circ} 16^{\prime} 00^{\prime} \mathrm{W}\right)\end{array}$ \\
\hline Phataria unifascialis & ICML-UNAM 2.18.103 & 17 & $\begin{array}{l}\text { Puerto Balandra, Baja California Sur, Mexico } \\
\left(24^{\circ} 31^{\prime} 48^{\prime \prime} \mathrm{N}, 110^{\circ} 31^{\prime} 15^{\prime \prime} \mathrm{W}\right)\end{array}$ \\
\hline Phataria unifascialis & ICML-UNAM 2.18.104 & 4 & $\begin{array}{l}\text { Bahía San Gabriel, Isla Espíritu Santo, Baja California Sur, } \\
\text { Mexico }\left(24^{\circ} 30^{\prime} 00^{\prime \prime} \mathrm{N}, 110^{\circ} 21^{\prime} 00^{\prime} \mathrm{W}\right) \text {. }\end{array}$ \\
\hline Phataria unifascialis & ICML-UNAM 2.18.105 & 15 & $\begin{array}{l}\text { La Gaviota, La Paz, Baja California Sur, Mexico } \\
\left(24^{\circ} 17^{\prime} 13^{\prime \prime} \mathrm{N}, 110^{\circ} 20^{\prime} 34^{\prime \prime} \mathrm{W}\right)\end{array}$ \\
\hline Phataria unifascialis & ICML-UNAM 2.18.106 & 10 & $\begin{array}{l}\text { El Embudo, Isla Espíritu Santo, Baja California Sur, Mexico } \\
\left(24^{\circ} 33^{\prime} 00^{\prime} \mathrm{N}, 110^{\circ} 22^{\prime} 00^{\prime} \mathrm{W}\right)\end{array}$ \\
\hline Phataria unifascialis & ICML-UNAM 2.18.107 & 2 & $\begin{array}{l}\text { Bahía Falsa, Baja California Sur, Mexico } \\
\left(24^{\circ} 20^{\prime} 00^{\prime} \mathrm{N}, 110^{\circ} 01^{\prime} 00^{\prime \prime} \mathrm{W}\right)\end{array}$ \\
\hline Phataria unifascialis & ICML-UNAM 2.18.108 & 2 & $\begin{array}{l}\text { Bahía Falsa, Baja California Sur, Mexico } \\
\left(24^{\circ} 20^{\prime} 00^{\prime} \mathrm{N}, 110^{\circ} 01^{\prime} 00^{\prime \prime} \mathrm{W}\right)\end{array}$ \\
\hline Phataria unifascialis & ICML-UNAM 2.18.109 & 1 & $\begin{array}{l}\text { Bahía Falsa, Baja California Sur, Mexico } \\
\left(24^{\circ} 20^{\prime} 00^{\prime} \mathrm{N}, 110^{\circ} 01^{\prime} 00^{\prime} \mathrm{W}\right)\end{array}$ \\
\hline Phataria unifascialis & ICML-UNAM 2.18.110 & 37 & $\begin{array}{l}\text { Puerto Balandra, Baja California Sur, Mexico } \\
\left(24^{\circ} 31^{\prime} 48^{\prime \prime} \mathrm{N}, 110^{\circ} 31^{\prime} 15^{\prime} \mathrm{W}\right)\end{array}$ \\
\hline Phataria unifascialis & ICML-UNAM 2.18.111 & 1 & $\begin{array}{l}\text { Bahía San Gabriel, Isla Espíritu Santo, Baja California Sur, } \\
\text { Mexico }\left(24^{\circ} 30^{\prime} 00^{\prime} \mathrm{N}, 110^{\circ} 21^{\prime} 00^{\prime} \mathrm{W}\right) \text {. }\end{array}$ \\
\hline Phataria unifascialis & ICML-UNAM 2.18.112 & 2 & $\begin{array}{l}\text { Barra de Pulmo, Baja California Sur, Mexico } \\
\left(23^{\circ} 24^{\prime} 05^{\prime \prime} \mathrm{N}, 109^{\circ} 32^{\prime} 34^{\prime} \mathrm{W}\right)\end{array}$ \\
\hline Phataria unifascialis & ICML-UNAM 2.18.113 & 6 & $\begin{array}{l}\text { El Corralito, La Paz, Baja California Sur, Mexico } \\
\left(24^{\circ} 35^{\prime} 00^{\prime \prime} \mathrm{N}, 110^{\circ} 30^{\prime} 00^{\prime} \mathrm{W}\right)\end{array}$ \\
\hline Phataria unifascialis & ICML-UNAM 2.18.114 & 2 & $\begin{array}{l}\text { Bahía San Gabriel, Isla Espíritu Santo, Baja California Sur, } \\
\text { Mexico }\left(24^{\circ} 30^{\prime} 00^{\prime \prime} \mathrm{N}, 110^{\circ} 21^{\prime} 00^{\prime} \mathrm{W}\right) \text {. }\end{array}$ \\
\hline Phataria unifascialis & ICML-UNAM 2.18.115 & 1 & $\begin{array}{l}\text { La Bonanza, La Paz, Baja California Sur, Mexico } \\
\left(24^{\circ} 30^{\prime} 00^{\prime} \mathrm{N}, 110^{\circ} 15^{\prime} 00^{\prime} \mathrm{W}\right)\end{array}$ \\
\hline Phataria unifascialis & ICML-UNAM 2.18.116 & 9 & $\begin{array}{l}\text { Bahía San Gabriel, Isla Espíritu Santo, Baja California Sur, } \\
\text { Mexico }\left(24^{\circ} 30^{\prime} 00^{\prime \prime} \mathrm{N}, 110^{\circ} 21^{\prime} 00^{\prime \prime} \mathrm{W}\right)\end{array}$ \\
\hline Phataria unifascialis & ICML-UNAM 2.18.117 & 2 & $\begin{array}{l}\text { Estero de los Cuates, Baja California Norte } \\
\left(31^{\circ} 50^{\prime} 00^{\prime \prime} \mathrm{N}, 116^{\circ} 50^{\prime} 00^{\prime \prime} \mathrm{W}\right)\end{array}$ \\
\hline Phataria unifascialis & ICML-UNAM 2.18.118 & 1 & $\begin{array}{l}\text { Los Frailes, La Paz, Baja California Sur, Mexico } \\
\left(23^{\circ} 23^{\prime} 32^{\prime \prime} \mathrm{N}, 109^{\circ} 25^{\prime} 00^{\prime} \mathrm{W}\right) \text {. }\end{array}$ \\
\hline
\end{tabular}


TABLE 1 (Continued)

\begin{tabular}{|c|c|c|c|}
\hline & Catalogue number & $\begin{array}{l}\text { Number of } \\
\text { specimens }\end{array}$ & Locality \\
\hline Phataria unifascialis & ICML-UNAM 2.18.119 & 18 & $\begin{array}{l}\text { El Embudo, Isla Espíritu Santo, Baja California Sur, Mexico } \\
\left(24^{\circ} 30^{\prime} 00^{\prime \prime} \mathrm{N}, 110^{\circ} 21^{\prime} 00^{\prime \prime} \mathrm{W}\right) .\end{array}$ \\
\hline Phataria unifascialis & ICML-UNAM 2.18.120 & 2 & $\begin{array}{l}\text { Los Islotes, Baja California Sur, Mexico } \\
\left(24^{\circ} 35^{\prime} 09^{\prime \prime} \mathrm{N}, 110^{\circ} 24^{\prime} 09^{\prime} \mathrm{W}\right)\end{array}$ \\
\hline Phataria unifascialis & ICML-UNAM 2.18 .121 & 1 & $\begin{array}{l}\text { Bahía San Carlos, Guaymas, Sonora, Mexico } \\
\left(27^{\circ} 58^{\prime} 56^{\prime} \mathrm{N}, 110^{\circ} 58^{\prime} 36^{\prime} \mathrm{W}\right) \text {. }\end{array}$ \\
\hline Phataria unifascialis & ICML-UNAM 2.18.122 & 3 & $\begin{array}{l}\text { Isla Espíritu Santo, Baja California Sur, Mexico } \\
\left(24^{\circ} 30^{\prime} 00^{\prime \prime} \mathrm{N}, 110^{\circ} 21^{\prime} 00^{\prime \prime} \mathrm{W}\right) \text {. }\end{array}$ \\
\hline Phataria unifascialis & ICML-UNAM 2.18.123 & 1 & $\begin{array}{l}\text { Puerto Libertad, Sonora, Mexico } \\
\left(24^{\circ} 54^{\prime} 14^{\prime \prime} \mathrm{N}, 112^{\circ} 41^{\prime} 27^{\prime \prime} \mathrm{W}\right)\end{array}$ \\
\hline Phataria unifascialis & ICML-UNAM 2.18.125 & 1 & $\begin{array}{l}\text { Los Islotes, Baja California Sur, Mexico } \\
\left(24^{\circ} 35^{\prime} 05^{\prime \prime} \mathrm{N}, 110^{\circ} 24^{\prime} 56^{\prime \prime} \mathrm{W}\right)\end{array}$ \\
\hline Phataria unifascialis & ICML-UNAM 2.18.126 & 1 & $\begin{array}{l}\text { Los Islotes, Baja California Sur, Mexico } \\
\left(24^{\circ} 35^{\prime} 05^{\prime \prime} \mathrm{N}, 110^{\circ} 24^{\prime} 56^{\prime \prime} \mathrm{W}\right)\end{array}$ \\
\hline Phataria unifascialis & ICML-UNAM 2.18.127 & 1 & $\begin{array}{l}\text { Los Islotes, Baja California Sur, Mexico } \\
\left(24^{\circ} 35^{\prime} 57^{\prime \prime} \mathrm{N}, 110^{\circ} 24^{\prime} 59^{\prime} \text { W). }\right.\end{array}$ \\
\hline Phataria unifascialis & ICML-UNAM 2.18.128 & 3 & $\begin{array}{l}\text { Los Islotes, Baja California Sur, Mexico } \\
\left(24^{\circ} 35^{\prime} 57^{\prime \prime} \mathrm{N}, 110^{\circ} 24^{\prime} 20^{\prime \prime} \mathrm{W}\right)\end{array}$ \\
\hline Phataria unifascialis & ICML-UNAM 2.18.130 & 21 & $\begin{array}{l}\text { El Coyote cove, La Paz, Baja California Sur, Mexico } \\
\left(24^{\circ} 10^{\prime} 00^{\prime} \mathrm{N}, 110^{\circ} 18^{\prime} 00^{\prime} \mathrm{W}\right) \text {. }\end{array}$ \\
\hline Phataria unifascialis & ICML-UNAM 2.18.139 & 1 & $\begin{array}{l}\text { Los Islotes, Baja California Sur, Mexico } \\
\left(24^{\circ} 35^{\prime} 57^{\prime} \mathrm{N}, 110^{\circ} 23^{\prime} 59^{\prime \prime} \mathrm{W}\right)\end{array}$ \\
\hline Phataria unifascialis & ICML-UNAM 2.18.151 & 1 & $\begin{array}{l}\text { South of Isla Santa Catalina, Baja California Sur, Mexico } \\
\left(25^{\circ} 30^{\prime} 54^{\prime} \mathrm{N}, 110^{\circ} 46^{\prime} 41^{\prime} \mathrm{W}\right) \text {. }\end{array}$ \\
\hline Phataria unifascialis & ICML-UNAM 2.18.152 & 1 & $\begin{array}{l}\text { Isla Las Ánimas, Baja California Sur, Mexico } \\
\left(25^{\circ} 06^{\prime} 52^{\prime} \mathrm{N}, 110^{\circ} 30^{\prime} 28^{\prime} \mathrm{W}\right)\end{array}$ \\
\hline Phataria unifascialis & ICML-UNAM 2.18.156 & 2 & $\begin{array}{l}\text { Bahía San Carlos, Guaymas, Sonora, Mexico } \\
\left(27^{\circ} 56^{\prime} 27^{\prime \prime} \mathrm{N}, 111^{\circ} 05^{\prime} 30^{\prime} \mathrm{W}\right) \text {. }\end{array}$ \\
\hline Phataria unifascialis & ICML-UNAM 2.18.157 & 3 & $\begin{array}{l}\text { El Peruano, Guaymas, Sonora, Mexico } \\
\left(27^{\circ} 54^{\prime} 30^{\prime \prime} \mathrm{N}, 110^{\circ} 58^{\prime} 11^{\prime \prime} \mathrm{W}\right)\end{array}$ \\
\hline Phataria mionactis & LACM 761.1 Holotype & 1 & La Plata Island, Ecuador (no lat long data). \\
\hline
\end{tabular}

was removed with regular household bleach $(\mathrm{NaClO})$, after that the individual skeletal plates were extracted from specimens or parts of them immersed, using regular household bleach, rinsed in tap water, dried, mounted on aluminium stubs and gold-coated (modified from Thuy \& Stöhr, 2016).

\section{RESULTS}

\section{Systematics}

\section{Phataria unifascialis (Gray, 1840) (Figs. 1-7)}

Linckia (Phataria) unifascialis Gray, 1840 Linckia bifascialis Gray, 1840
Linckia unifascialis Gray, 1840

Ophidiaster suturalis Müller \& Troschel, 1842

Original description (Gray, 1840): Rays trigonal, tapering, back with three rows of flat ossicula; sides with a single abroad band of pores; rather more than three times as long as broad.

Emended description: Five rays, tapering, papular areas only in the abactinal surface, the papular areas are in one longitudinal row along the arm, and also have two small rows of papular areas at both sides of the main row. Three longitudinal rows of carinal plates along the arm. Both surfaces are cover by granules, in 

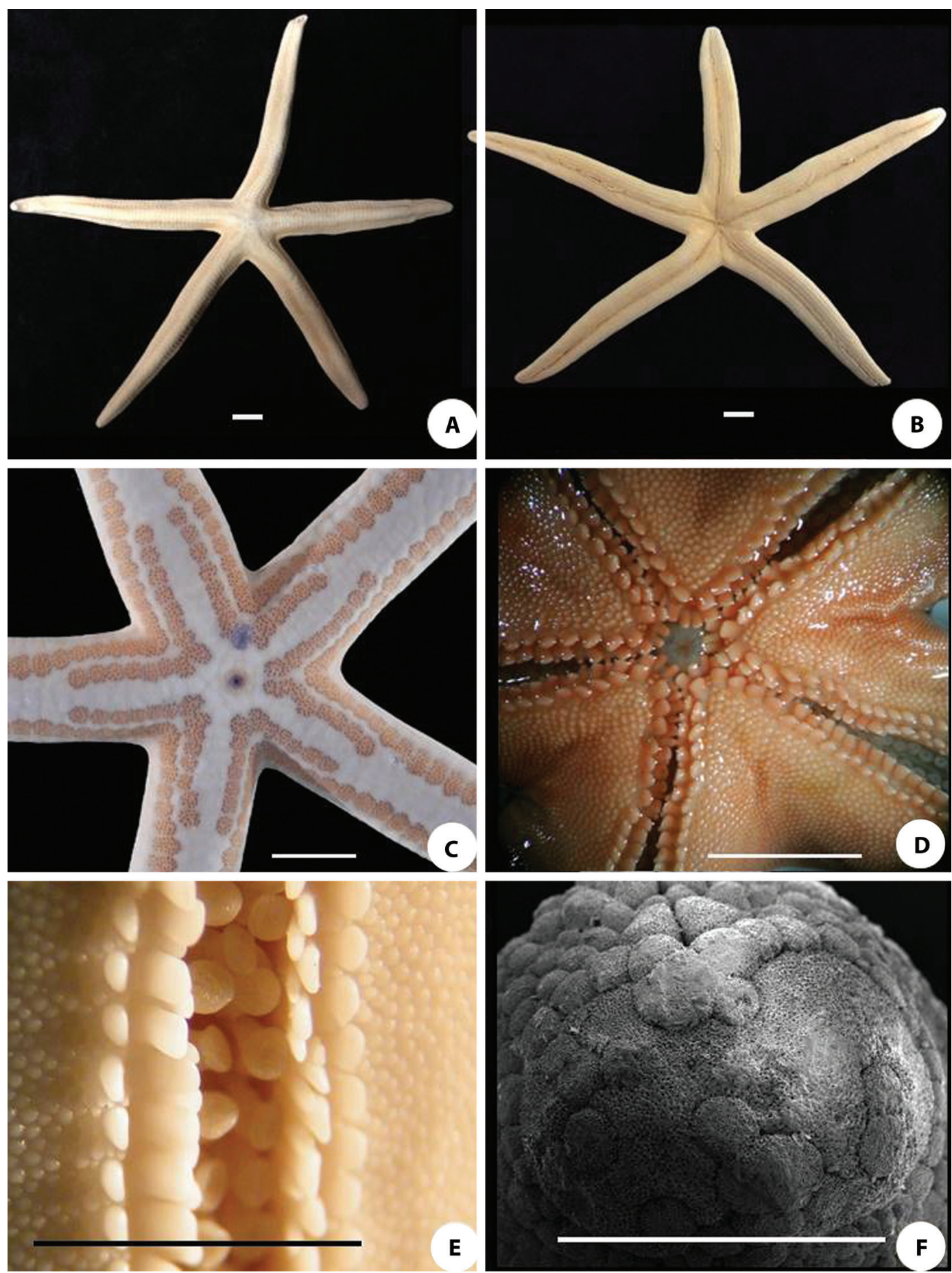

Fig. 1. Phataria unifascialis. A) Abactinal view; B) Actinal view; C) Abactinal disc; D) Actinal disc; E) Actinal arm; F) Terminal plate; (A,B,C and D scale bar $=1 \mathrm{~cm}$; E and $\mathrm{F}$ scale bar $=0.5 \mathrm{~cm})$.

Fig. 1. Phataria unifascialis. A) Vista abactinal; B) Vista actinal; C) Disco abactinal; D) Disco actinal; E) Brazo actinal; F) Placa terminal; (A,B,C y D escala $=1 \mathrm{~cm}$; E y F escala $=0.5 \mathrm{~cm})$. 


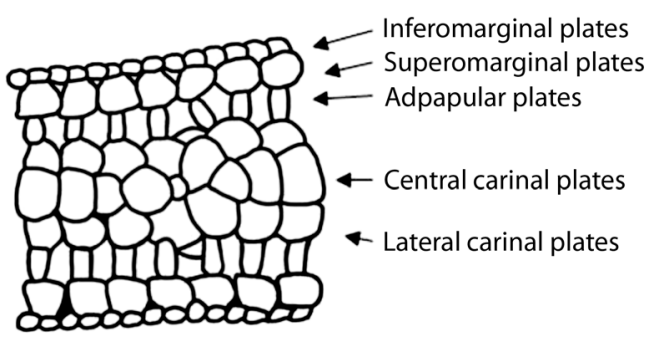

Fig. 2. Phataria unifascialis. Skeleton of the arm in abactinal view.

Fig. 2. Phataria unifascialis. Vista abactinal del esqueleto del brazo.

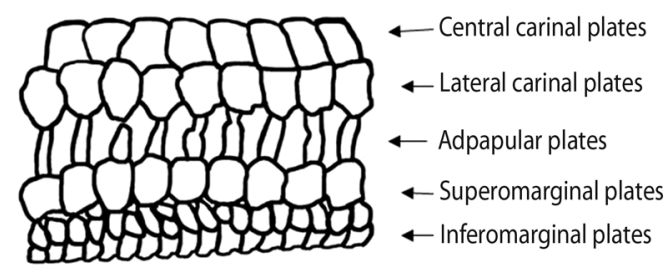

Fig. 3. Phataria unifascialis. Skeleton of the arm in a lateral view.

Fig. 3. Phataria unifascialis. Vista lateral del esqueleto del brazo.

the abactinal one there are small granules in the papular areas and bigger ones on the abactinal plates. The actinal surface with smaller, rounded granules with little space between them. The anus is located in the center of the disc; only one rounded madreporite with radial striations. Only one row of ambulacral spines; such spines are wider in the base and tapering (Fig. 1).

Morphology of the skeletal ossicles. The abactinal area is composed of carinal plates, adpapular plates and superomarginal plates (Fig. 2, Fig. 4 and Fig. 6). The actinal area is composed of inferomarginal plates, actinal plates, adambulacral plates and ambulacral furrow plates (Fig. 3, Fig. 5 and Fig. 7).

Type locality: "Bay of Caracas, West Columbia" (data from holotype label). But there is any locality in the Pacific Ocean with this name.
Type material: NHM 1938.5.12.4 (Natural History Museum, London).

Geographic distribution: Eastern Pacific. Mexico, El Salvador, Costa Rica, Panama, Colombia, Ecuador and Peru (Pérez-Ruzafa, A., Alvarado, J. J., Solís-Marín, F. A., Hérnandez, J. C., Morata, A., Marcos, C., ... \& Barraza, E, 2013).

Bathymetric distribution: From 0 and 50 m (Pérez-Ruzafa et al., 2013).

\section{DISCUSSION}

The original description made by Gray (1840) did not include the basic information to indetify $P$. unifascialis as a separated and independent genus of Ophidiasteridae, instead the specie was included in the genus Linckia (Gray, 1840). Gray's original description can be practically used to describe various genus or species within the family (i.e. Pharia, Phataria, Narcissia, Linckia, etc). The diagnosis presented in this work has the main taxonomical characters to unequivocally can identify the specimens belonging to this species. There are three main contributions in this new emended diagnosis: 1) the papular areas are distributed in one longitudinal row along the arm, and two small rows can reach half or less the main row at both sides of the main longitudinal papular row; 2) there are three longitudinal rows of carinal plates along each arm, and 3) there is only one row of ambulacral spines that are wider in the base and tapering. Currently the genus Phataria has only two valid species: Phataria unifascialis (Gray, 1840) and Phataria mionactis Ziesenhenne, 1942. The specie Phataria mionactis, which has a restricted distribution to the Galapagos Islands, is distinguished by the presence of two continuous rows of papular areas at both sides of the main row (Fig. 8); the carinal plates are in an irregular arrangement, smaller than those in $P$. unifascialis. The abactinal plates are convex in P. mionactis and flat in P. unifascialis (see Figs. 2 and 8). 


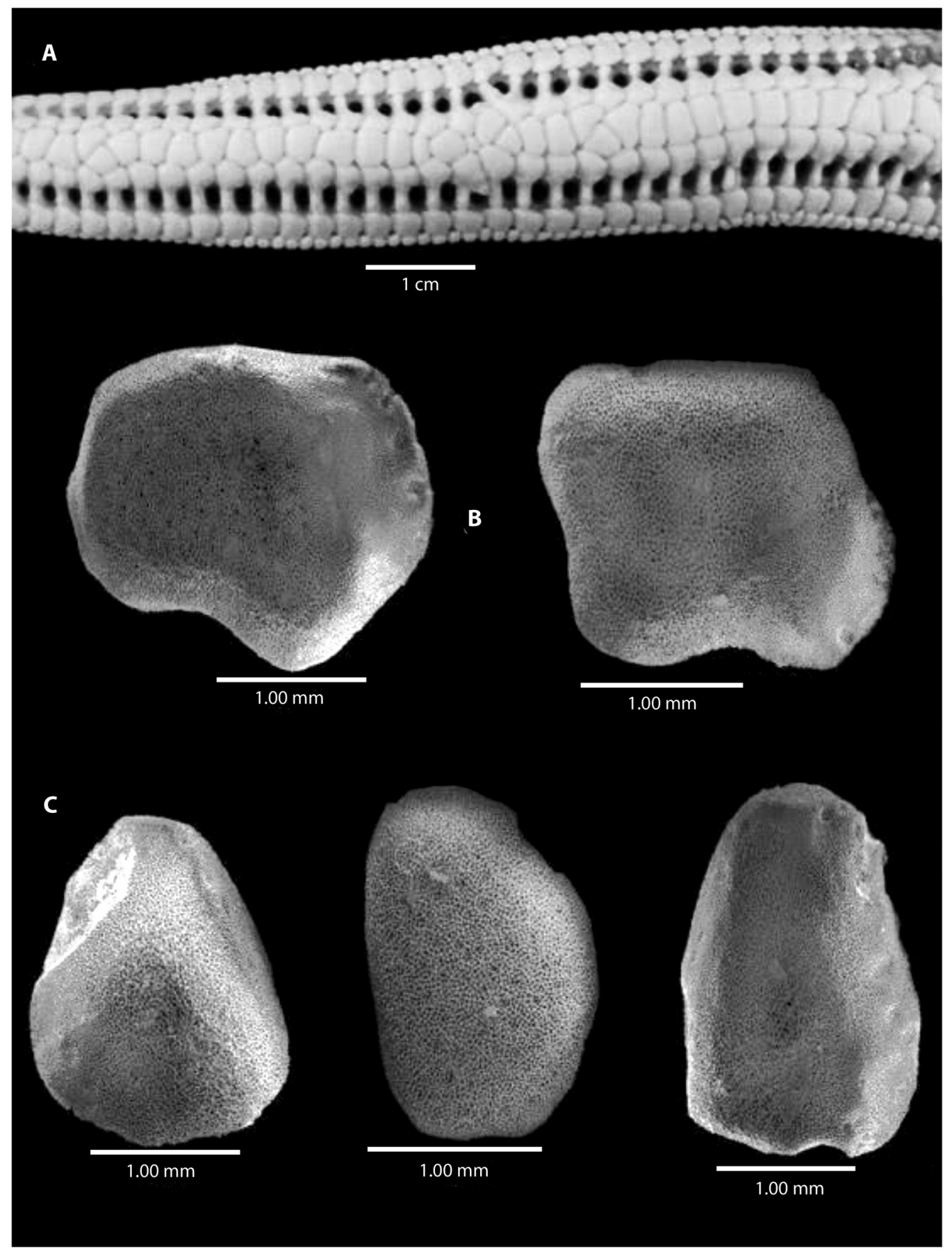

Fig. 4. Phataria unifascialis. A) Abactinal view of a duneded arm; B) Lateral carinal plates; C) Central carinal plates. Fig. 4. Phataria unifascialis. A) Vista abactinal de un brazo desnudo; B) Placas carinales laterales; C) Placas carinales centrales. 


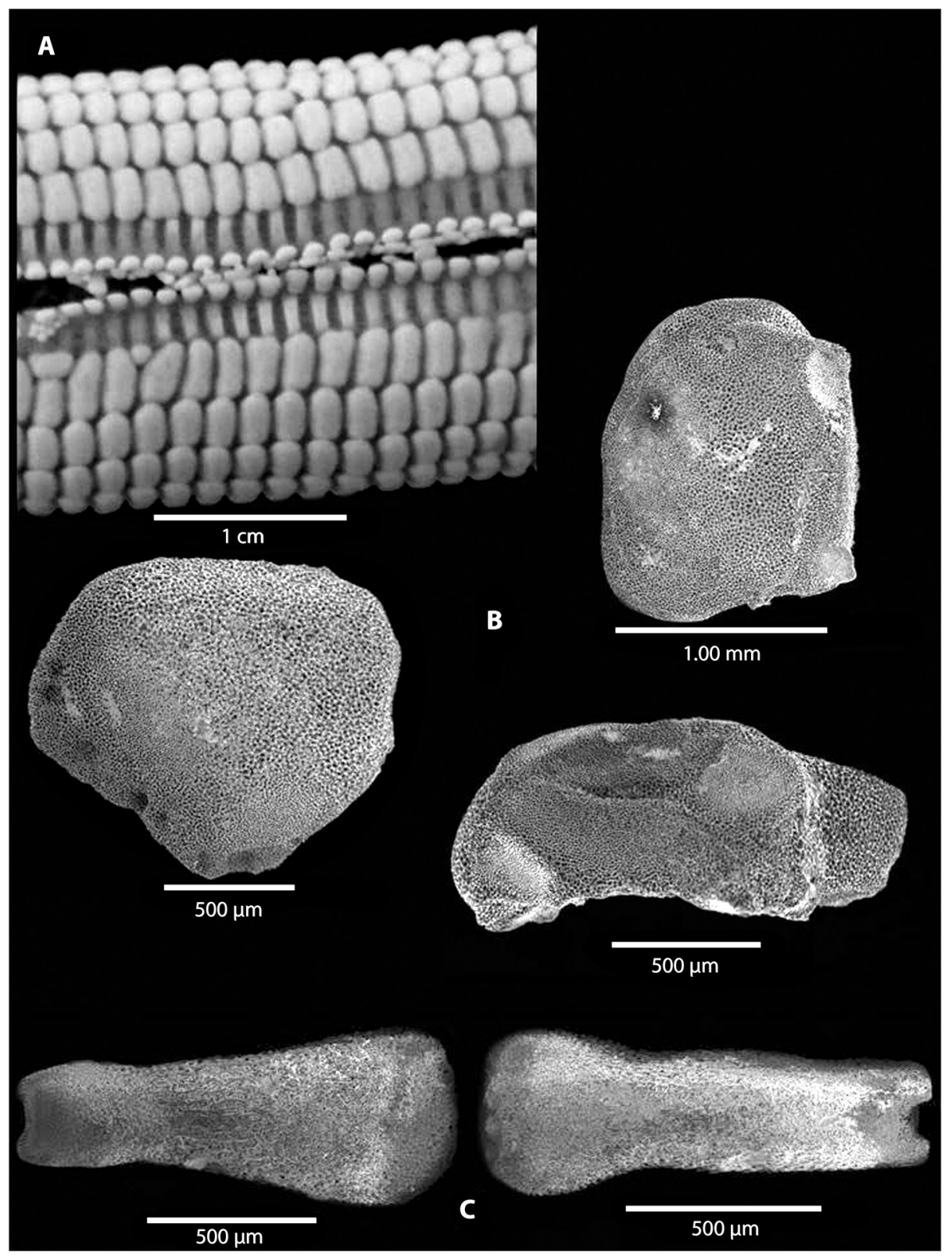

Fig. 5. Phataria unifascialis. A) Abactinal view of a duneded arm; B) Adambulacral plates; C) Adambulacral spines. Fig. 5. Phataria unifascialis. A) Vista abactinal de un brazo desnudo; B) Placas adambulacrales; C) Espinas adambulacrales. 


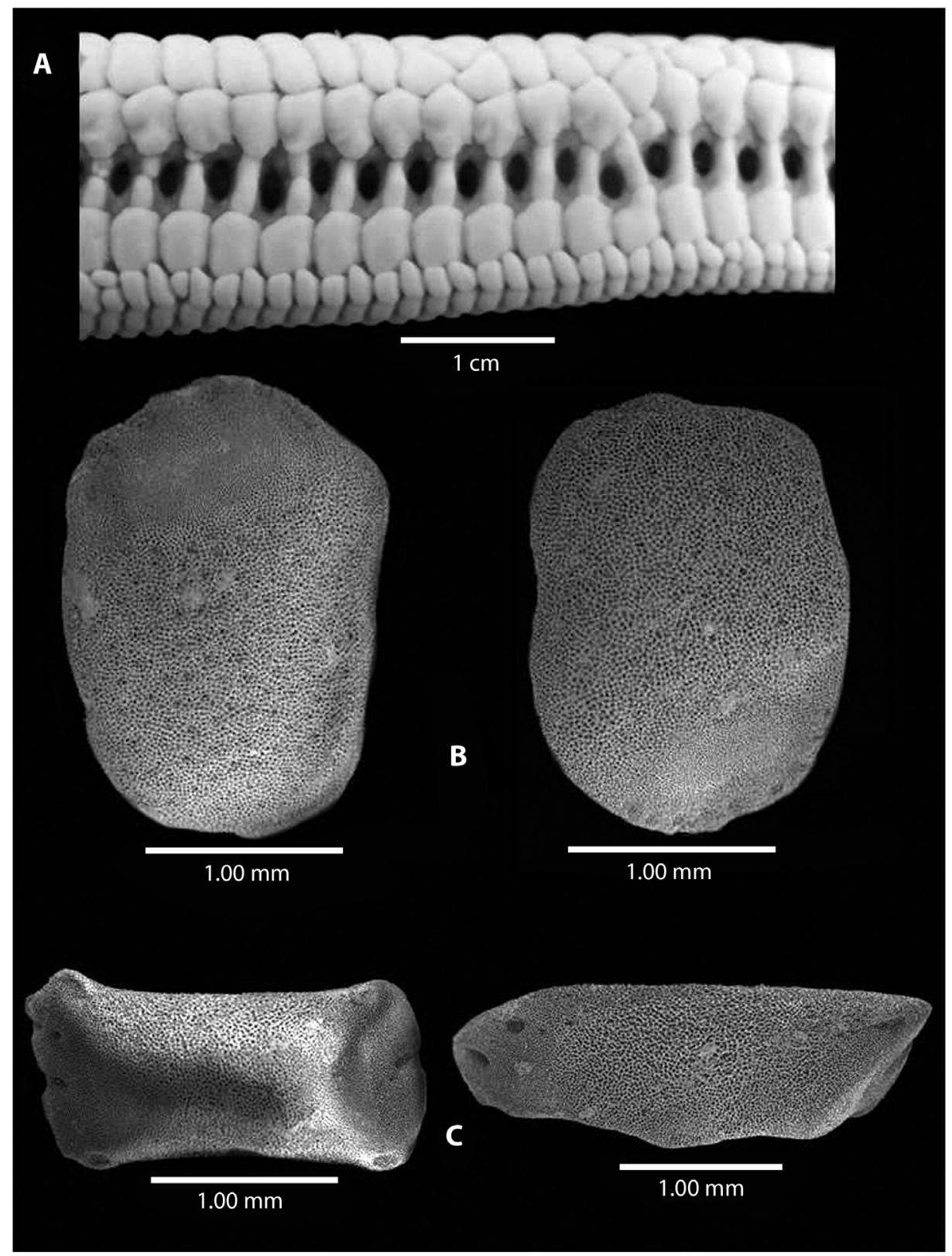

Fig. 6. Phataria unifascialis. A) Lateral view of a duneded arm; B) Superomarginal plates; C) Adpapular plates.

Fig. 6. Phataria unifascialis. A) Vista lateral de un brazo desnudo; B) Placas súperomarginales; C) Placas adpapulares. 


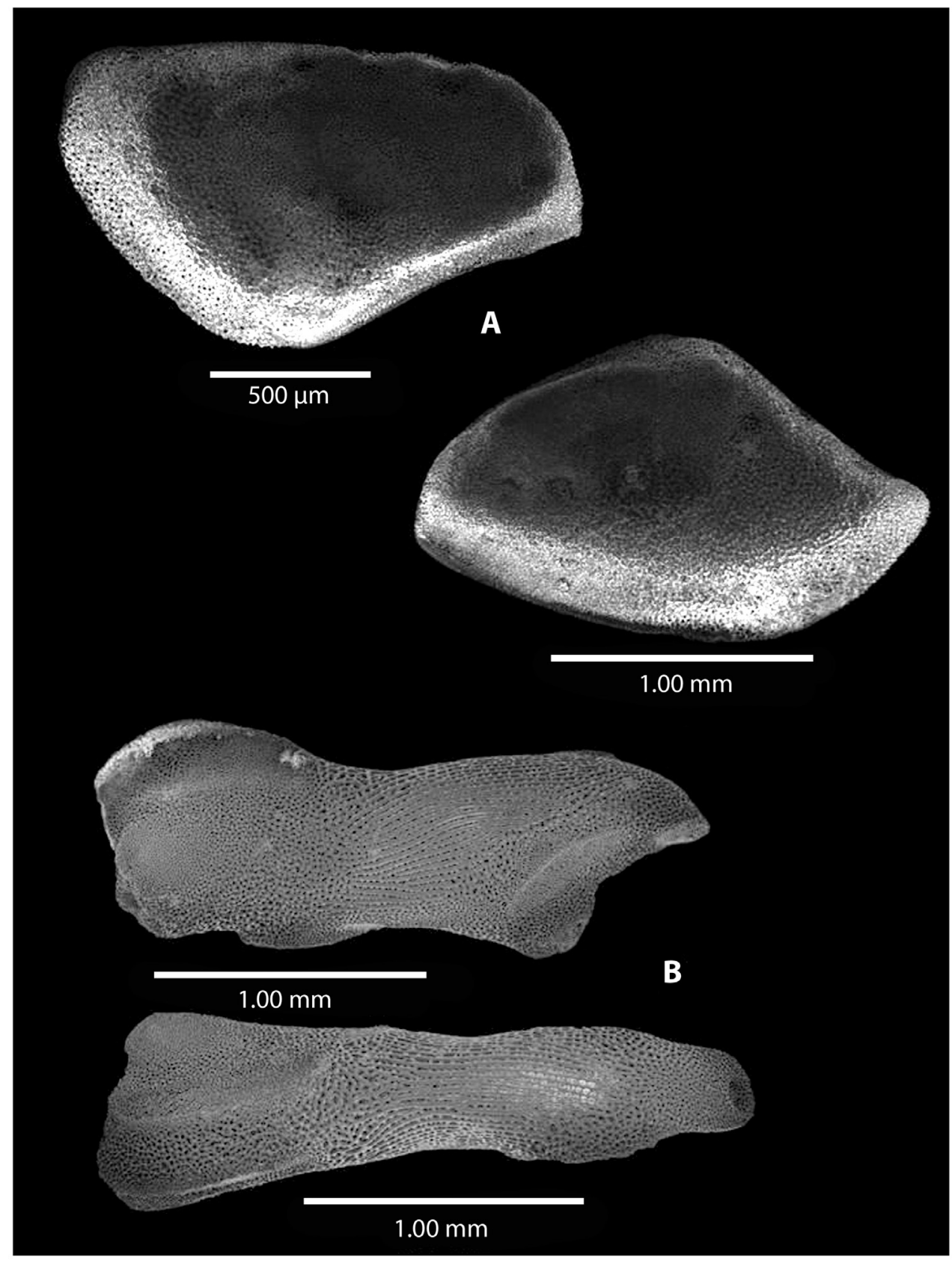

Fig. 7. Phataria unifascialis. Holotype LACM 761.1. A) Actinal plates; B) Plates of the ambulacral furrow. Fig. 7. Phataria unifascialis. Holotipo LACM 761.1. A) Placas actinales; B) Placas del surco ambulacral. 


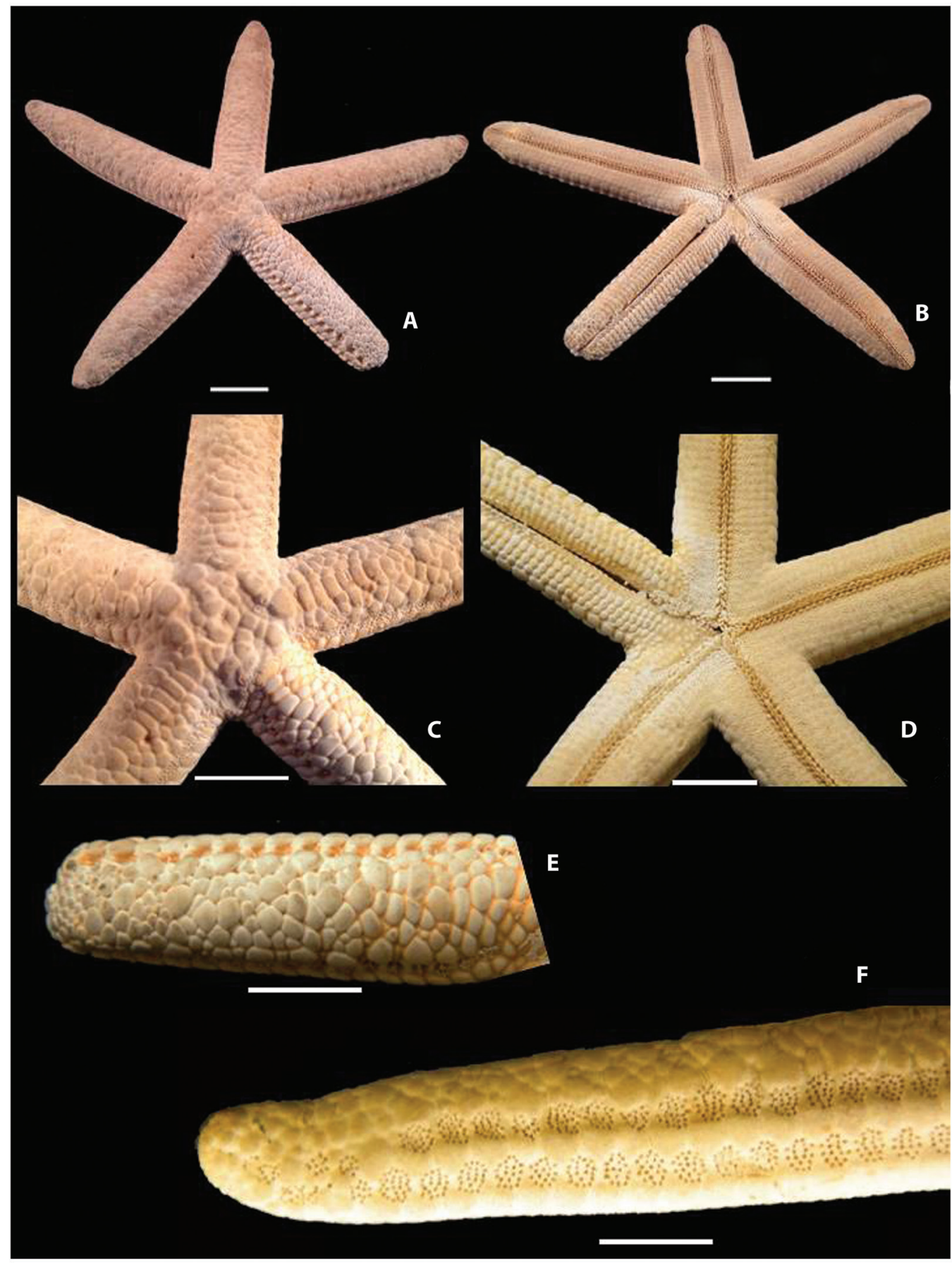

Fig. 8. Phataria mionactis. A) Abactinal view; B) Actinal view; C) Abactinal disc; D) Actinal disc; E) Abactinal arm; F) Longitudinal rows of papular areas; (Scale bar $=1 \mathrm{~cm})$.

Fig. 8. Phataria mionactis. A) Vista abactinal; B) Vista actinal; C) Disco abactinal; D) Disco actinal; E) Brazo abactinal; F) Hileras longitudinales de áreas papulares; $($ Escala $=1 \mathrm{~cm})$. 
The type locality of $P$. unifascialis was not mentioned by Gray (1840), nevertheless the locality in the holoype label reads: "Bay of Caracas, West Columbia, Venezuela" (Andrew Cabrinovic pers. comm.). Ziesenhenne (1937), mentions that the type locality of the species was "Bay of Caracas, west coast of Colombia". There is no locality in the Colombian Pacific under that name and because the species has a known distribution on the American Pacific Ocean coasts, the type locality is not in Venezuela since this country is located in the Atlantic Ocean. More research is needed to determine the original type locality of $P$. unifascialis.

The use of plate morphology in Asteroidea identification is higly recommended as presented by Turner and Dearborn (1972). More efforts need to be done in order to use these taxonomic characters, even though type material is so limited and fragile. Whenever material is available, skeletal morphology studies are suggested.

\section{ACKNOWLEDGMENTS}

To Maria Berenit Mendoza Garfias (Laboratorio de Microscopía Electrónica y Fotografía de la Biodiversidad, Instituto de Biología, UNAM) for her technical support with the SEM photos. To Andrew Cabrinovic (Aquatic Invertebrates Division, The Natural History Museum, UK) for the information about the holotype.

\section{RESUMEN}

Phataria unifascialis (Echinodermata: Asteroidea) de la costa este del Océano Pacífico: redescripción y morfología del esqueleto. La estrella de mar Phataria unifascialis está ampliamente distribuida sobre fondos rocosos entre 0 y $50 \mathrm{~m}$ de profundidad en Pacífico Este. La descripción original de P. unifascialis realizada por Gray en 1840 fue breve e inexacta en algunos aspectos importantes; actualmente tenemos más información sobre la morfología de esta especie. Este trabajo incluye una descripción detallada de $P$. unifascialis añadiendo por primera vez imágenes SEM y descripción de sus placas esqueléticas.

Palabras clave: Asteroidea; Ophidiasteridae; Phataria unifascialis; redescripción; SEM; Pacífico Este.

\section{REFERENCES}

Alvarado, J. J., \& Solís-Marín, F. A. (2013). Echinoderm research and diversity in Latin America (pp. 1-9). Springer Berlin Heidelberg.

Gray, J. E. (1840). A synopsis of the genera and species of the class Hypostoma (Asterias Linnaeus). Annals and Magazine of Natural History 6: 175-184 and 275-290.

Morgan, M. B., \& Cowles, D. L. (1997). The effects of temperature on the behaviour and physiology of Phataria unifascialis (Gray) (Echinodermata, Asteroidea) Implications for the species' distribution in the Gulf of California, Mexico. Journal of experimental marine biology and ecology, 208(1-2), 13-27.

Mortensen, T., \& Mortensen, T. (1921). Studies of the development and larval forms of echinoderms. Gad.

Pérez-Ruzafa, A., Alvarado, J. J., Solís-Marín, F. A., Hernández, J. C., Morata, A., Marcos, C., ... \& Barraza, E. (2013). Latin America echinoderm biodiversity and biogeography: Patterns and affinities. In Echinoderm research and diversity in Latin America (pp. 511-542). Springer Berlin Heidelberg.

Thuy, B., \& Stöhr, S. (2016). A new morphological phylogeny of the Ophiuroidea (Echinodermata) accords with molecular evidence and renders microfossils accessible for cladistics. PloS one, 11(5), e0156140.

Turner, R. L., \& Dearborn, J. H. (1972). Skeletal morphology of the mud star, Ctenodiscus crispatus (Echinodermata: Asteroidea). Journal of Morphology, 138(2), 239-262.

Sladen, W. P. (1889). Asteroidea. Report on the Asteroidea collected during the voyage of HMS 'Challenger'during the years 1873Á76. Report on the Scientific Results of the Voyage of HMS Challenger. Zoology, 30.

Ziesenhenne, F. C. (1937). The Templeton Crocker Expedition. X. Echinoderms from the west coast of Lower California, the Gulf of California and Clarion Island. Zoologica, NY, 22, 209-239. 